Pacific Journal of Mathematics

ON THE GROWTH OF FUNCTIONS HAVING POLES OR
ZEROS ON THE POSITIVe REAL AXIS 


\title{
ON THE GROWTH OF FUNCTIONS HAVING POLES OR ZEROS ON THE POSITIVE REAL AXIS
}

\author{
H. D. BRUNK
}

1. Introduction. Let $\Gamma$ denote the boundary of a region $\Delta$ containing a right half of the real axis, and $f(z)$ a function holomorphic in $\Delta$ except possibly for simple poles on the positive real axis. Through the residue theorem, the integral

$$
\frac{1}{2 \pi i} \int_{\Gamma} f(z) e^{-z s} d z
$$

makes correspond to $f(z)$ a Dirichlet series whose coefficients are the residues of $f(z)$ at its poles. It is the purpose of this paper to exhibit some immediate applications, using this familiar device, of the theory of asymptotic Dirichlet series to a study of the growth of functions with poles of bounded order, or zeros of at least a certain order, on the positive real axis. Let the poles, or zeros, occur at points $z=\lambda_{n}$, where $0<\lambda_{n} \uparrow \propto$. Theorems $2 \mathrm{~A}$ and $2 \mathrm{~B}$, in $\S 3$, relate the growth of leading coefficients in the Laurent developments about the points $z=\lambda_{n}$ to the growth of $f(z)$ in $\Delta$. Theorems $3 A$ and $3 B$, in $\$ 4$, apply to functions of exponential type in the right half-plane outside $\Delta$, satisfying much weaker growth conditions in $\Delta$. Theorem $3 \mathrm{~A}$ may be thought of as stating that such a function, with a specified rapidity of decrease on the imaginary axis, is holomorphic if it does not have poles of too great order; while Theorems $3 \mathrm{~A}$ and $3 \mathrm{~B}$ together may be regarded as saying that if such a function has "zeros" of minimum order too great for its growth on the imaginary axis, poles counting as negative zeros, then it vanishes identically. (The emphasis on this aspect of Theorem $3 \mathrm{~A}$ and this interpretation of the uniqueness conclusions of Theorems $3 A$ and $3 B$ were suggested by the referee.)

2. Definitions, the fundamental inequality, and properties of the function $C(z)$. Let $\left\{\lambda_{n}\right\} \quad(n=1,2, \ldots)$ be a sequence of positive numbers, strictly increasing without bound $\left(0<\lambda_{n} \uparrow \infty\right)$, such that

$$
\limsup _{n \rightarrow \infty} \frac{n}{\lambda_{n}}=D^{\bullet}<\infty
$$

Received February 9, 1953.

Pacific J. Math. 4 (1954), 1 - 19 
Let

$$
\Lambda_{n}^{*}=\prod_{k \neq n}\left|\frac{\lambda_{k}^{2}}{\lambda_{k}^{2}-\lambda_{n}^{2}}\right|
$$

Let $\Delta$ denote a region in the complex $z$-plane $(z=x+i y)$ bounded by

$$
y=g^{+}(x), y=-g^{-}(x), \text { and } x=a\left(0 \leq a<\lambda_{1}\right) \text {, }
$$

where $g^{+}(x)$ and $g^{-}(x)$ are defined for $x \geq a$, positive for $x>a$, continuous, bounded, and satisfy Lipschitz conditions: there exists a constant $K$ such that

$$
\begin{aligned}
& \left|g^{+}(x+\Delta x)-g^{+}(x)\right|<K|\Delta x|, \\
& \left|g^{-}(x+\Delta x)-g^{-}(x)\right|<K|\Delta x|,
\end{aligned}
$$

for $x>a, x+\Delta x>a$. Denote by $\Gamma$ the complete boundary of $\Delta$, by $\Gamma^{+}$the part of $\Gamma$ in the upper half-plane, and by $\Gamma^{-}$the part of $\Gamma$ in the lower half-plane.

Let $\left\{M_{n}\right\}(n=1,2, \ldots)$ be a sequence of positive constants, and set

$$
p(\sigma)=\sup _{n \geq 1}\left(\lambda_{n} \sigma-\log M_{n}\right) .
$$

Following the notation introduced by Mandelbrojt [6], we shall say that the sequence $\left\{M_{n}\right\}$ has the uniqueness property $(U)$ if, for some positive number $\eta$,

$$
\int^{\infty} p(\sigma) e^{-\eta \sigma} d \sigma=\infty
$$

(The absence of the lower limit indicates that the relation is to hold provided the lower limit is sufficiently large.) If, in particular,

$$
\liminf _{n \rightarrow \infty} \frac{\log M_{n}}{\lambda_{n}}<\infty,
$$

then $p(\sigma) \equiv \propto$ for $\sigma$ sufficiently large, so that (U) holds. If

$$
\lim _{n \rightarrow \infty} \frac{\log M_{n}}{\lambda_{n}}=\infty
$$

let the sequence $\left\{M_{n}^{c}\right\}(n=1,2, \ldots)$ be defined so that $\log M_{n}^{c}$ is the greatest monotone, nondecreasing, convex function of $n$ which is not greater than $\log M_{n}$. 
Mandelbrojt has shown $[6$; cf. also $7, \S 1.8, \mathrm{III}-\mathrm{V}]$ that condition (U) is equivalent to

$(\mathrm{U})^{\prime}$

$$
\sum^{\infty}\left(\frac{M_{n}^{c}}{M_{n+1}^{c}}\right)^{\eta /\left(\lambda_{n+1}-\lambda_{n}\right)}\left[\lambda_{n+1}-\lambda_{n}\right]=\infty
$$

for some positive $\eta$.

If $n$ is a positive integer, the sums $\sum_{k=1}^{m} a_{k} \exp \left(-\lambda_{k} s\right)(m \geq n)$ are said to represent a function $F(s)$ with logarithmic precision $p_{n}(\sigma)$ in a region containing points with arbitrarily large real part if for $s$ in that region and for $x$ sufficiently large,

$$
\inf _{m \geq n} \sup _{\sigma \geq x}\left|F(s)-\sum_{k=1}^{m} a_{k} \exp \left(-\lambda_{k} s\right)\right| \leq \exp \left(-p_{n}(x)\right) .
$$

Mandelbrojt has proved $[7, \S 3.7]$ a theorem relating the growth of the function $F(s)$ to the growth of the coefficients $a_{n}$. This theorem contains as a very special case the following theorem, sufficient for the needs of the present paper.

THEOREM 1. Let the sequence $\left\{\lambda_{n}\right\}$ satisfy (1). Let $\sigma_{0}$ and $\sigma_{1}$ be real numbers, and $R$ a positive number greater than $D^{*}$, such that $F(s)(s=\sigma+i t)$ is holomorphic in the half-plane $\sigma \geq \sigma_{0}$, and continuable analytically throughout the strip

$$
S:|t| \leq \pi R, \sigma \geq \sigma_{1}-R
$$

For each positive $h$, let there exist a constant $A_{h}$ such that the sums $\sum_{k=1}^{m}$ $a_{k} \exp \left(-\lambda_{k} s\right)(m \geq n)$ represent $F(s)$ in $\sigma \geq \sigma_{0},|t| \leq h$ with logarithmic precision $p_{n}(\sigma)-A_{h}$ satisfying

$$
\int^{\infty} p_{n}(\sigma) e^{-\eta \sigma} d \sigma=\infty
$$

for some positive $\eta$.

Then there exists a constant $B$, depending on $R$, such that

$$
\left|a_{n}\right| \leq B \lambda_{n} \Lambda_{n}^{*} M\left(\sigma_{1}, R\right) \exp \left(\lambda_{n} \sigma_{1}\right),
$$

where $M\left(\sigma_{1}, R\right)$ denotes the maximum of $|F(s)|$ in the circle $\left|s-\sigma_{1}\right| \leq-R$. 
Let $C(z)$ denote the entire function $[1, \mathrm{p} .267] \prod_{n=1}^{\infty}\left(1-z^{2} / \lambda_{n}^{2}\right)$. The following well-known properties of $C(z)$ will be useful in extending to functions with poles of bounded order and to holomorphic functions the results obtained for functions with simple poles by the device described in the introductory paragraph.

I. For every positive number $\epsilon$, and for $z$ sufficiently large [1, p. 267],

$$
|C(z)|<\exp \left[\left(D^{\bullet}+\epsilon\right)|z|\right] \text {. }
$$

II. If

$$
\inf _{n \geq 1}\left(\lambda_{n+1}-\lambda_{n}\right)>0,
$$

and $\epsilon(r)$ is a nonincreasing, positive function of $r$ such that

$$
\inf _{r>0} \frac{\log \epsilon(r)}{r}>-\infty
$$

then there exists a constant $K>0$ such that

$$
|C(z)|>\exp (-K|z|)
$$

for $z$ exterior to every circle centered at a point $z= \pm \lambda_{n}$ and having radius $\epsilon\left(\lambda_{n}\right)$.

Proof of II. This property of $C(z)$ follows easily from inequalities of Mandelbrojt on the numbers $\Lambda_{n}^{*}[7, \S 3.3$, II ]. If

$$
\inf _{n}\left(\lambda_{n+1}-\lambda_{n}\right) \geq h \text { and } \sup _{n} \frac{n}{\lambda_{n}} \leq C,
$$

then there exists a constant $K_{1}$ depending on $C$ and on $h$ but otherwise independent of the particular sequence $\left\{\lambda_{n}\right\}$, such that

$$
\log \Lambda_{n}^{*}=\log \prod_{k \neq n}\left|\frac{\lambda_{k}^{2}}{\lambda_{k}^{2}-\lambda_{n}^{2}}\right|<K_{1} \lambda_{n} ;
$$

hence

$$
\log \prod_{k \neq n}\left|1-\frac{\lambda_{n}^{2}}{\lambda_{k}^{2}}\right|>-K_{1} \lambda_{n}
$$


Let $z=r e^{i \theta}=x+i y$, and suppose (without loss of generality, since $C(z)$ is even in $z$ ) that $z$ lies in the half-plane $x \geq 0$, exterior to each circle, centered at a point $\lambda_{k}$ and of radius $\epsilon\left(\lambda_{k}\right)$ :

$$
\left|z-\lambda_{k}\right|>\epsilon\left(\lambda_{k}\right)
$$

Fix $z$, and, if $r \geq \lambda_{1}$, choose $n$ so that $\lambda_{n} \leq r<\lambda_{n+1}$. Then

$$
\prod_{k \neq n}\left|1-\frac{z^{2}}{\lambda_{k}^{2}}\right| \geq \prod_{k \neq n}\left|1-\frac{r^{2}}{\lambda_{k}^{2}}\right|
$$

since this inequality holds for corresponding factors. Define a sequence $\left\{\lambda_{k}^{*}\right\}$ :

$$
\lambda_{k}^{*}=\lambda_{k} \text { for } k<n, \lambda_{n}^{*}=r_{s} \lambda_{n+j}^{*}=\lambda_{n+j+1} \quad(i=1,2, \cdots) .
$$

For the sequence $\left\{\lambda_{k}^{*}\right\}$ we have also

$$
\inf _{k}\left(\lambda_{k+1}^{*}-\lambda_{k}^{*}\right) \geq h \text { and } \sup _{k} \frac{k}{\lambda_{k}^{*}} \leq C
$$

so that

$$
\prod_{k \neq n}\left|1-\frac{\lambda_{n}^{* 2}}{\lambda_{k}^{* 2}}\right|=\prod_{k \neq n, n+1}\left|1-\frac{r^{2}}{\lambda_{k}^{2}}\right|>\exp \left(-K_{1} r\right) .
$$

From (10) and (11) it follows that

$$
|C(z)|=\prod_{k=1}^{\infty}\left|1-\frac{z^{2}}{\lambda_{k}^{2}}\right|>\left|1-\frac{z^{2}}{\lambda_{n}^{2}}\right|\left|1-\frac{z^{2}}{\lambda_{n+1}^{2}}\right| \exp \left(-K_{1} r\right) .
$$

If $\lambda_{n+1} \geq 2 r$, then

$$
\left|1-\frac{z^{2}}{\lambda_{n+1}^{2}}\right| \geq\left|1-\frac{r^{2}}{\lambda_{n+1}^{2}}\right| \geq \frac{3}{4}
$$

whereas if $\lambda_{n+1}<2 r$, then 


$$
\left|1-\frac{z^{2}}{\lambda_{n+1}^{2}}\right|=\frac{\left|\lambda_{n+1}^{2}-z^{2}\right|}{\lambda_{n+1}^{2}} \geq \frac{\epsilon\left(\lambda_{n+1}\right)}{\lambda_{n+1}}>\frac{\epsilon(2 r)}{2 r} .
$$

Also

$$
\left|1-\frac{z^{2}}{\lambda_{n}^{2}}\right|=\frac{\left|\lambda_{n}^{2}-z^{2}\right|}{\lambda_{n}^{2}} \geq \frac{\epsilon\left(\lambda_{n}\right)}{\lambda_{n}}
$$

Hence

$$
|C(z)| \geq \frac{\epsilon\left(\lambda_{n}\right)}{\lambda_{n}} \exp \left(-K_{1} r\right) \min \left[\frac{3}{4}, \frac{\epsilon(2 r)}{2 r}\right]
$$

If $r<\lambda_{t}$, we define

$$
\lambda_{1}^{*}=r, \lambda_{k}^{*}=\lambda_{k} \quad(k=2,3, \cdots)
$$

and find that

$$
\prod_{k=2}^{\infty}\left|1-\left(\frac{\lambda_{1}^{*}}{\lambda_{k}^{*}}\right)^{2}\right|=\prod_{k=2}^{\infty}\left|1-\left(\frac{r}{\lambda_{k}}\right)^{2}\right|>\exp \left(-K_{1} r\right)
$$

so that

$$
|C(z)|=\prod_{k=1}^{\infty}\left|1-\left(\frac{z}{\lambda_{k}}\right)^{2}\right|>\frac{\left|z^{2}-\lambda_{1}^{2}\right|}{\lambda_{1}^{2}} \exp \left(-K_{1} r\right) \geq \frac{\epsilon\left(\lambda_{1}\right)}{\lambda_{1}} \exp \left(-K_{1} r\right) .
$$

From (8) and (12) it then follows that there exists a constant $K$ such that

$$
|C(z)|>e^{-K r}=e^{-K|z|} .
$$

III. If $\lim _{n \rightarrow \infty} n / \lambda_{n}=D>0$, and $\theta \neq n \pi$ for every positive integer $n$, then $[1, \mathrm{p} .279]$

$$
\lim _{r \rightarrow \infty}\left[\log \left|C\left(r e^{i \theta}\right)\right|\right] / r=\pi D|\sin \theta| .
$$




\section{Growth theorems.}

LEMMA 1. Let $f(z)$ satisfy the following hypotheses:

(14) $f(z)$ is holomorphic in $\Delta$ except for simple poles with residues $a_{k}$ at points $z=\lambda_{k}(k=1,2, \cdots)$, and is continuous on the boundary $\Gamma$ of $\Delta$ :

(15) there exists a constant a such that $f(z)=O\left(e^{a x}\right)$ as $z \longrightarrow \infty$ on $\Gamma$;

(16) there exist positive constants $M_{n}$, and rectifiable curves $\gamma_{n}$, of uniformly bounded length, joining $\Gamma^{-}$and $\Gamma^{+}$, such that $x \geq \lambda_{n}$ and $|f(z)|^{n}<M_{n}$ for $z$ on $\gamma_{n}(n=1,2, \cdots)$.

Then

(17) the integral $(1 / 2 \pi i) \int_{\Gamma} f(z) e^{-z s} d z \quad(s=\sigma+i t)(\Gamma$ being traversed from $z=\propto$ along $\Gamma^{+}$and out along $\Gamma^{-}$to $z=\propto$ again) converges for $\sigma>\propto$ to a function $F(s)$ holomorphic in $\sigma>\alpha$;

(18) if

$$
\liminf _{n \rightarrow \infty} \frac{\log M_{n}}{\lambda_{n}}<\infty
$$

then there exists a sequence $\left\{n_{k}\right\}$ of positive integers such that for $\sigma$ sufficiently large, $\sum_{j=1}^{n_{k}} a_{j} \exp \left(-\lambda_{j} s\right)$ converges to $F(s)$ as $k \rightarrow \infty$; if, further,

$$
\inf _{n \geq 1}\left(\lambda_{n+1}-\lambda_{n}\right)>0
$$

then the series $\sum_{1}^{\infty} a_{j} \exp \left(-\lambda_{j} s\right)$ converges to $F(s)$ for $\sigma$ sufficiently large; (19) if

$$
\lim _{n \rightarrow \infty} \frac{\log M_{n}}{\lambda_{n}}=\infty,|t| \leq h, \text { and } \sigma \geq \alpha+\delta \text {, }
$$

where $h, \delta$ are arbitrary positive numbers, then there exists a constant $K=K(h, \delta)$ such that

$$
\left|F(s)-\sum_{k=1}^{n} a_{k} \exp \left(-\lambda_{k} s\right)\right|<K M_{n} \exp \left(-\lambda_{n} \sigma\right) \quad(n=1,2, \ldots) .
$$


Proof. Let $\Delta_{n}$ denote that one of the two regions bounded by $\Gamma$ and by $\gamma_{n}$ which contains $\lambda_{1}, \lambda_{2}, \ldots, \lambda_{n}$, and let $\Gamma_{n}$ denote its boundary. By the residue the orem,

$$
\frac{1}{2 \pi i} \int_{\Gamma_{n}} f(z) e^{-z s} d z=\sum_{k=1}^{n} a_{k} \exp \left(-\lambda_{k} s\right) .
$$

Denote by $\Gamma_{n}^{+}$and $\Gamma_{n}^{-}$those parts of $\Gamma^{+}$and $\Gamma^{-}$(cf. page 2 ) respectively which do not belong to $\Gamma_{n}$. By (15), there exists a positive constant $C_{1}$ such that

$$
\left|\frac{1}{2 \pi i} \int_{\Gamma_{n}^{+}} f(z) e^{-z s} d z\right| \leq C_{1} \int_{\lambda_{n}}^{\infty} e^{a x} e^{-(\sigma x-|t| k)}|d z|
$$

where $k$ is a bound on $g^{+}(x)$ and on $g^{-}(x)$. The function $g^{+}(x)$ satisfies the Lipschitz condition (3), so that if $|t| \leq h$, there exists a positive constant $C_{2}=C_{2}(h)$ such that

$$
\left|\frac{1}{2 \pi i} \int_{\Gamma_{n}^{+}} f(z) e^{-z s} d z\right| \leq C_{2} \int_{\lambda_{n}}^{\infty} e^{-(\sigma-\alpha) x} d x .
$$

Finally, if also $\sigma \geq a+\delta$, there exists a positive constant $C_{3}=C_{3}(h, \delta)$, such that

$$
\left|\frac{1}{2 \pi i} \int_{\Gamma_{n}^{+}} f(z) e^{-z s} d z\right| \leq C_{3} \exp \left(\alpha \lambda_{n}\right) \exp \left(-\lambda_{n} \sigma\right)
$$

It follows that

$$
\frac{1}{2 \pi i} \int_{\Gamma^{+}} f(z) e^{-z s} d z
$$

converges to a function $F^{+}(s)$, holomorphic in $\sigma>\alpha$. Similarly, if $|t| \leq h$ and $\sigma \geq \alpha+\delta$, there exists a positive constant $C_{4}=C_{4}(h, \delta)$, such that

$$
\left|\frac{1}{2 \pi i} \int_{\Gamma_{n}^{-}} f(z) e^{-z s} d z\right| \leq C_{4} \exp \left(\alpha \lambda_{n}\right) \exp \left(-\lambda_{n} \sigma\right) \text {. }
$$

The integral

$$
\frac{1}{2 \pi i} \int_{\Gamma^{-}} f(z) e^{-z s} d z
$$


therefore also converges, to a function $F^{-}(s)$ holomorphic in $\sigma>\alpha$, and we have $F(s)=F^{+}(s)+F^{-}(s)$; the proof of $(17)$ is complete.

We have also

$$
\begin{aligned}
\left|\frac{1}{2 \pi i} \int_{\gamma_{n}} f(z) e^{-z s} d z\right| & \leq \frac{M_{n}}{2 \pi} \int_{\gamma_{n}} e^{-(\sigma x-t y)}|d z| \\
& \leq \frac{L M_{n}}{2 \pi} \exp (k|t|) \exp \left(-\lambda_{n} \sigma\right),
\end{aligned}
$$

where $L$ is a uniform bound on the lengths of the curves $\gamma_{n}$, and $k$ is a bound on $g^{+}(x)$ and on $g^{-}(x)$. Thus if $|t| \leq h$, there exists a positive constant $C_{5}=C_{5}(h)$, such that

$$
\left|\frac{1}{2 \pi i} \int_{\gamma_{n}} f(z) e^{-z s} d z\right| \leq C_{5} M_{n} \exp \left(-\lambda_{n} \sigma\right) .
$$

Suppose now that

$$
\liminf _{n \rightarrow \infty} \frac{\log M_{n}}{\lambda_{n}}<\infty
$$

Then from (20), (21), (22), and (23) it follows that there exists a constant $C_{6}$ and a sequence $\left\{n_{k}\right\}$ of positive integers, such that

$$
\lim _{k \rightarrow \infty} \sum_{j=1}^{n_{k}} a_{j} \exp \left(-\lambda_{j} \delta\right)=F(s)
$$

for $\sigma$ sufficiently large. If in addition

$$
\inf _{n \geq 1}\left(\lambda_{n+1}-\lambda_{n}\right)>0,
$$

it follows from a result of Mandelbrojt $[7, \S 3.10, \mathrm{~V}]$ that $\sum_{1}^{\infty} a_{k} \exp \left(-\lambda_{k} s\right)$ converges to $F(s)$ for $\sigma$ sufficiently large. This completes the proof of (18).

If, on the contrary,

$$
\lim _{n \rightarrow \infty} \frac{\log M_{n}}{\lambda_{n}}=\infty
$$


then there exists a positive constant $C_{7}$, such that $\exp \left(\alpha \lambda_{n}\right)<C_{7} M_{n}(n=1$, $2, \ldots)$. It then follows from (20), (21), (22), and (23) that if $|t| \leq h$, and $\sigma \geq \alpha+\delta$, there exists a positive constant $K=K(h, \delta)$ such that

$$
\left|F(s)-\sum_{1}^{n} a_{k} \exp \left(-\lambda_{k} s\right)\right| \leq K M_{n} \exp \left(-\lambda_{n} \sigma\right) \quad(n=1,2, \cdots) .
$$

This completes the proof of (19) and of Lemma 1.

THEOREM 2. Let $f(z)$ satisfy the hypotheses of Lemma 1, and let the sequence $\left\{M_{n}\right\}$ have the uniqueness property (U). Then for every positive number $\delta$,

$$
\left|a_{n}\right|=o\left(\lambda_{n} \Lambda_{n}^{*} \exp \left[\left(\alpha+\delta+D^{\bullet}\right) \lambda_{n}\right]\right) \text { as } n \rightarrow \infty \text {. }
$$

If further

$$
\inf _{n \geq 1}\left(\lambda_{n+1}-\lambda_{n}\right)>0
$$

then the series $\sum a_{k} \exp \left(-\lambda_{k} s\right)$ converges to $F(s)$ for $\sigma$ sufficiently large, which implies that

$$
\limsup _{n \rightarrow \infty} \frac{\log \left|a_{n}\right|}{\lambda_{n}}<\infty
$$

Thus it is clear that if

$$
\inf _{n \geq 1}\left(\lambda_{n+1}-\lambda_{n}\right)>0,
$$

and the residues $a_{k}$ increase so rapidly that

$$
\lim _{n \rightarrow \infty} \frac{\log \left|a_{n}\right|}{\lambda_{n}}=\infty
$$

then the bounds $M_{n}$ of the function $f(z)$ on the curves $\gamma_{n}$ must increase so rapidly that the sequence $\left\{M_{n}\right\}$ fails to satisfy the uniqueness condition (U), that is, so rapidly that for every positive number $\eta$, the series

$$
\sum\left(\frac{M_{n}^{c}}{M_{n+1}^{c}}\right)^{n /\left(\lambda_{n+1}-\lambda_{n}\right)}\left[\lambda_{n+1}-\lambda_{n}\right]
$$


converges.

Proof of Theorem 2. If

$$
\liminf _{n \rightarrow \infty} \frac{\log M_{n}}{\lambda_{n}}<\infty
$$

it follows from equations $(20),(21),(22)$, and (23) that if

$$
0 \leq \delta^{\circ}<\delta, \quad \sigma \geq \alpha+\delta^{\circ}, \text { and }|t| \leq h,
$$

then there exist a constant $C=C\left(h, \delta^{\prime}\right)$, a constant $b$, and a sequence $\left\{n_{k}\right\}$ such that

$$
\left|F(s)-\sum_{j=1}^{n_{k}} a_{j} \exp \left(-\lambda_{j} s\right)\right|<C \exp \left[-\lambda_{n_{k}}(\sigma-b)\right]
$$

It follows then from the definition (4) that for each $\delta^{\circ}$ and $h$, and each positive integer $n$, the sums $\sum_{j=1}^{m} a_{j} \exp \left(-\lambda_{j} s\right)(m \geq n)$ represent $F(s)$ in $\sigma \geq \alpha+\delta^{\prime}$, $|t| \leq h$, with infinite logarithmic precision. The inequality (6), with $\sigma_{1}=\alpha+$ $\delta^{\circ}+D^{\circ}$, then yields $(24)$. If

$$
\lim _{n \rightarrow \infty} \frac{\log M_{n}}{\lambda_{n}}=\infty
$$

then (19) and Theorem 1 imply the conclusion (24). Mandelbrojt has shown $[7, \S 3.3$, III $]$ that if

$$
\inf _{n \geq 1}\left(\lambda_{n+1}-\lambda_{n}\right)>0
$$

then

$$
\sup \frac{\log \Lambda_{n}^{*}}{\lambda_{n}}<\infty,
$$

which, with (24), implies (25) (cf. $[1$, p.4]), and completes the proof of Theorem 2.

THEOREM 2A. Let $p$ be a positive integer, and let $f(z)$ satisfy the hypotheses of Theorem 2, except that the poles at points $z=\lambda_{n}$ need not be simple, 
but none is of order greater than p. Let $a_{n,-p}$ denote the coefficient of the leading term $a_{n,-p} /\left(z-\lambda_{n}\right)^{p}$ in the Laurent development of $f(z)$ about $z=\lambda_{n}(n=$ $1,2, \cdots)$. Let the curve $\gamma_{n}$ referred to in hypothesis (16) of Lemma 1 lie entirely between $x=\lambda_{n}$ and $x=\lambda_{n}+d$ for some fixed positive constant $d$ $(n=1,2, \cdots)$. Then for every positive number $\delta$,

$$
\left|a_{n,-p}\right|=o\left(\lambda_{n}^{p} \Lambda_{n}^{* p} \exp \left[\left(\alpha+\delta+D^{\bullet}\right) \lambda_{n}\right]\right) \quad \text { as } n \rightarrow \infty \text {. }
$$

If further

$$
\inf _{n \geq 1}\left(\dot{\lambda}_{n+1}-\lambda_{n}\right)>0,
$$

then

$$
\limsup _{n \rightarrow \infty} \frac{\log \left|a_{n,-p}\right|}{\lambda_{n}}<\infty .
$$

The added hypothesis on the curves $\gamma_{n}$ is not very stringent. In fact, since these curves have uniformly bounded length, and since $\gamma_{n}$ lies to the right of the line $x=\lambda_{n}$, there exists such a constant $d$ as is required, if only each of the curves $\gamma_{n}$ has at least one point within a fixed distance independent of $n$ from the corresponding point $z=\lambda_{n}$.

THE OREM 2B. Let $p$ be a positive integer, and let $f(z)$ satisfy the hypotheses of Theorem 2, except that $f(z)$ is holomorphic in $\Delta$, with zeros at points $z=\lambda_{n}$ of order at least $p$. Let $a_{n, p}$ denote the coefficient of the leading term $a_{n, p}\left(z-\lambda_{n}\right)^{p}$ in the Taylor series development of $f$ about $z=\lambda_{n} \quad(n=1$, $2, \cdots)$. Let the boundary of $\Delta$ satisfy the further condition that

$$
\liminf _{r \rightarrow \infty} \frac{\log g^{+}(r)}{r}>-\infty \text {, and } \liminf _{r \rightarrow \infty} \frac{\log g^{-}(r)}{r}>-\infty \text {. }
$$

Let there exist a nonincreasing, positive function $\epsilon(r)$ of $r$ such that

$$
\inf _{r>0} \frac{\log \epsilon(r)}{r}>-\infty
$$

and such that the curves $\gamma_{n}$ referred to in hypothesis (16) of Lemma 1 do not penetrate circles of radius $\epsilon\left(\lambda_{n}\right)$ about the points $z=\lambda_{n}$. Further, let there exist a positive constant $d$ such that $|z|<\lambda_{n}+d$ for $z$ on $\gamma_{n}$. Then for 
every positive number $\delta$,

$$
\left|a_{n, p}\right|=o\left(\lambda_{n}^{-p} \Lambda_{n}^{*-p} \exp \left[\left(\alpha+D^{\bullet}+\delta\right)\right] \lambda_{n}\right) \text { as } n \rightarrow \infty \text {. }
$$

If further

$$
\inf _{n \geq 1}\left(\lambda_{n+1}-\lambda_{n}\right)>0,
$$

then

$$
\limsup _{n \rightarrow \infty} \frac{\log \left|a_{n, p}\right|}{\lambda_{n}}<\propto
$$

Proof of Theorems $2 \mathrm{~A}$ and $2 \mathrm{~B}$. If

$$
f^{*}(z)=f(z)[C(z)]^{j-1},
$$

where $j=p$ for Theorem $2 \mathrm{~A}$, and $j=-p$ for Theorem $2 \mathrm{~B}$, then $f^{*}(z)$ has simple poles at points $z=\lambda_{n}$. The coefficient of the leading term in the Taylor's expansion of $C(z)$ about the point $z=\lambda_{n}$ has absolute value $2 / \lambda_{n} \Lambda_{n}^{*}$, so that the residue of $f^{*}(z)$ at $z=\lambda_{n}$ has absolute value

$$
\left|a_{n}^{*}\right|=\left|a_{n,-j}\right|\left(\frac{2}{\lambda_{n} \Lambda_{n}^{*}}\right)^{j-1}
$$

It follows from properties I and II of the function $C(z)$ that $f^{*}(z)$ satisfies hypothesis (15) of Lemma 1, and that, since, for $z$ on $\gamma_{n},|z|-\lambda_{n}$ has a bound independent of $n$, there exist positive constants $A$ and $B$ such that

$$
\left|f^{*}(z)\right|<M_{n}^{*}=A M_{n} \exp \left(B \lambda_{n}\right) \text { for } z \text { on } \gamma_{n} \text {. }
$$

One readily verifies that if the sequence $\left\{M_{n}\right\}$ has the uniqueness property (U), so also does the sequence $\left\{M_{n}^{*}\right\}$. Indeed,

$$
\begin{aligned}
p^{*}(\sigma) & =\sup _{n \geq 1}\left(\lambda_{n} \sigma-\log M_{n}^{*}\right) \\
& =\sup _{n \geq 1}\left\{\lambda_{n}(\sigma-B)-\log M_{n}-\log A\right\}=p(\sigma-B)-\log A .
\end{aligned}
$$

Thus the function $f^{*}(z)$ satisfies the hypotheses of Theorem 2. Conclusion (24) applied to the coefficients $a_{n}^{*}$ yields (26) and (28). Further, the ratio 
$\left(\log \Lambda_{n}^{*}\right) / \lambda_{n}$ is bounded below; for if $L=\sup _{k \geq 1} k / \lambda_{k}$, then

$$
\begin{aligned}
\frac{1}{\Lambda_{n}^{*}}=\prod_{k \neq n}\left|1-\frac{\lambda_{n}^{2}}{\lambda_{k}^{2}}\right| \leq \prod_{k=1}^{\infty}\left(1+\frac{\lambda_{n}^{2}}{\lambda_{k}^{2}}\right) \leq \prod_{k=1}^{\infty}\left(1+\frac{L^{2} \lambda_{n}^{2}}{k^{2}}\right) \\
=\frac{\sin \pi i L \lambda_{n}}{\pi i L \lambda_{n}} \leq \frac{1}{\pi L \lambda_{1}} \exp \left(\pi L \lambda_{n}\right) .
\end{aligned}
$$

Therefore (25) implies (29). Also, as was seen in the proof of Theorem 2, if

$$
\inf _{n \geq 1}\left(\lambda_{n+1}-\lambda_{n}\right)>0,
$$

then $\left(\log \Lambda_{n}^{*}\right) / \lambda_{n}$ is bounded above, so that (25) implies (27). This completes the proof of Theorems $2 \mathrm{~A}$ and $2 \mathrm{~B}$.

4. Functions of exponential type in the right half-plane outside $\Delta$. The following lemma gives conditions on $f(z)$ sufficient in order that the function $F(s)$ defined in Lemma 1 shall be continuable analytically throughout a horizontal strip.

Let $D$ denote the complement in the half-plane $x>0$ of the region $\Delta$, and $\bar{D}$ its closure.

LEMMA 2. Let $f(z)$ satisfy the hypotheses of Lemma 1 , and also the following:

$$
f(z) \text { is holomorphic in } D, \text { continuous in } \bar{D} \text {; }
$$

(32) there exists a positive constant $\beta_{1}$ such that $\mid f($ iy $) \mid=O\left(\exp \left(-\beta_{1} y\right)\right)$ as $y \rightarrow \infty$;

(33) there exists a positive constant $\beta_{2}$ such that $\mid f($ iy $) \mid=O\left(\exp \left(\beta_{2} y\right)\right)$ as $y \rightarrow-\infty$.

Then the function $F(s)$ defined in Lemma 1 is holomorphic in the strip $-\beta_{2}<$ $t<\beta_{1}$, and uniformly bounded in every closed interior strip.

Proof. Let $K^{+}$denote the curve extending from $z=\infty$ along the $y$-axis to the origin, and along the $x$-axis to $x=a$, and $K^{-}$the curve traversed from $x=a$ 
along the $x$-axis to the origin, then along the lower half of the $y$-axis. Let

$$
F_{1}^{+}(s)=\frac{1}{2 \pi i} \int_{K^{+}} f(z) e^{-z s} d z, F_{1}^{-}(s)=\frac{1}{2 \pi i} \int_{K^{-}} f(z) e^{-z s} d z,
$$

and

$$
F_{1}(s)=F_{1}^{+}(s)+F_{1}^{-}(s)=\frac{1}{2 \pi i} \int_{i \infty}^{-i \infty} f(z) e^{-z s} d z
$$

By hypotheses (32) and (33), there exist constants $A^{+}$and $A^{-}$such that

$$
\left|\frac{1}{2 \pi i} \int_{i \infty}^{0} f(z) e^{-z s} d z\right| \leq A^{+} \int_{0}^{\infty} \exp \left(-\beta_{1} y\right) \exp (t y) d y,
$$

and

$$
\left|\frac{1}{2 \pi i} \int_{0}^{-i \infty} f(z) e^{-z s} d z\right| \leq A^{-} \int_{-\infty}^{0} \exp \left(\beta_{2} y\right) \exp (t y) d y .
$$

The functions $F_{1}^{+}(s)$ and $F_{1}^{-}(s)$ are therefore holomorphic in the half-planes $t<\beta_{1}$ and $t>-\beta_{2}$, respectively, and their sum $F_{1}(s)$ is holomorphic in the strip $-\beta_{2}<t<\beta_{1}$; and, for every positive number $\epsilon, F_{1}(s)$ is uniformly bounded in the strip $-\beta_{2}+\epsilon<t<\beta_{1}-\epsilon$. Let $C_{r}^{+}$denote that part in $D$ and above the $x$-axis of the circle of radius $r$ centered at the origin, traversed in a clockwise direction. Let $G_{r}^{+}$denote the boundary of the region above the $x$-axis, bounded by the $x$-axis, $\Gamma^{+}, C_{r}^{+}$, and the positive $y$-axis. Define similarly $C_{r}^{-}$and $G_{r}^{-}$below the $x$-axis. The contours $G_{r}^{+}$and $G_{r}^{-}$enclose parts of $D$, in which $f(z)$ is, by hypothesis, holomorphic, so that

$$
\frac{1}{2 \pi i} \int_{G_{r}^{+}} f(z) e^{-z s} d z \equiv \frac{1}{2 \pi i} \int_{G_{r}^{-}} f(z) e^{-z s} d z \equiv 0
$$

The integral

$$
\frac{1}{2 \pi i} \int_{\Gamma^{+}} f(z) e^{-z s} d z
$$

defines the function $F^{+}(s)$, which was seen in Lemma 1 to be holomorphic in the half-plane $\sigma>\alpha$. From (34) and the definitions of $F^{+}(s)$ and $F_{1}^{+}(s)$ it follows that 


$$
2 \pi i\left[F_{1}^{+}(s)-F^{+}(s)\right]=\int_{i \infty}^{i r}+\int_{C_{r}^{+}}-\int_{\Gamma_{r}^{+}} f(z) e^{-z s} d z
$$

where $\Gamma_{r}^{+}$is traversed along $\Gamma^{+}$from $z=\infty$ to the intersection of $\Gamma^{+}$with $C_{r}^{+}$, and where the path of integration for the first integral lies on the $y$-axis. It has already been seen that the first and last integrals tend to 0 as $r \rightarrow \infty$, for $s$ in the quarter-plane $\sigma>\alpha, t<\beta_{1}$. To show $F_{1}^{+}(s) \equiv F(s)$, it is therefore sufficient to show that, for $s$ on a continuum in the region $\sigma>\alpha, t<\beta_{1}$, the second integral,

$$
\int_{C_{r}^{+}} f(z) e^{-z s} d z
$$

tends to 0 as $r \rightarrow \infty$. By hypothesis (31), there exists a constant $B$ such that

$$
\begin{array}{r}
\left|\int_{C_{r}^{+}} f(z) e^{-z s} d z\right| \leq B e^{\alpha r}\left[\int_{0}^{\pi / 4}+\int_{\pi / 4}^{\pi / 2} r \exp \{-r(\sigma \cos \theta-t \sin \theta)\} d \theta\right] \\
\leq B r e^{a r}\left[e^{r|t|} \int_{0}^{\pi / 4} \exp (-\sigma r \cos \theta) d \theta+\int_{\pi / 4}^{\pi / 2} \exp (r t \sin \theta) d \theta\right]
\end{array}
$$

if $\sigma>0$, and

$$
\left|\int_{C_{r}^{+}} f(z) e^{-z s} d z\right| \leq \frac{\pi}{4} B r e^{\alpha r}\left[\exp (r|t|) \exp \left(\frac{-r \sigma}{\sqrt{2}}\right)+\exp \left(\frac{r t}{\sqrt{2}}\right)\right]
$$

if $\sigma>0, t<0$. Thus if

$$
t=-t_{0}<-\alpha \sqrt{2} \text {, and } \sigma>\sqrt{2}\left(\alpha+t_{0}\right) \text {, }
$$

then the integral approaches 0 as $r \longrightarrow \infty$. It follows that $F_{1}^{+}(s) \equiv F^{+}(s)$. Similarly, for $s$ on a suitable horizontal line in the upper half-plane and for $\sigma$ sufficiently large we have

$$
\frac{1}{2 \pi i} \int_{C_{r^{-}}^{-}} f(z) e^{-z s} d z \rightarrow 0 \text { as } r \rightarrow \infty \text {, }
$$

so that $F_{1}^{-}(s) \equiv F^{-}(s)$. Hence $F(s) \equiv F_{1}(s)$, and this completes the proof of Lemma 2.

THEOREM 3A. Let $f(z)$ satisfy the hypotheses of Theorem 2A and in 
addition $(30)-(33)$, with $\beta_{1}>\pi p D^{*}, \beta_{2}>\pi p D^{*}$. Then $a_{n,-k}=0,1 \leq k \leq p$ $(n=1,2, \cdots)$, so that $f(z)$ is holomorphic in $x>0$. If, further,

$$
\lim _{n \rightarrow \infty} \frac{n}{\lambda_{n}}=D=D^{\bullet}>0,
$$

then $f(z) \equiv 0$.

THEOREM 3B. Let $f(z)$ satisfy the hypotheses of Theorem $2 \mathrm{~B}$ and in addition $(30)-(33)$, except that $\beta_{1}>-\pi p D^{\circ}, \beta_{2}>-\pi p D^{\circ}$. Suppose further that

$$
\lim _{n \rightarrow \infty} \frac{n}{\lambda_{n}}=D=D^{\bullet}>0
$$

Then $f(z) \equiv 0$.

Proof of Theorems $3 \mathrm{~A}$ and $3 \mathrm{~B}$. As in the proof of Theorems $2 \mathrm{~A}$ and $2 \mathrm{~B}$, set

$$
f^{*}(z)=f(z)[C(z)]^{j-1},
$$

where $j=p$ for Theorem $3 \mathrm{~A}$ and $j=-p$ for Theorem 3B. It has been shown in the proofs of Theorems $2,2 \mathrm{~A}$, and $2 \mathrm{~B}$, that for each pair of positive numbers $\delta$ and $h$, the associated function $F^{*}(s)$ is represented in $\sigma \geq \alpha+\delta,|t| \leq h$ by the sums

$$
\sum_{k=1}^{m} a_{k}^{*} \exp \left(-\lambda_{k} s\right)
$$$$
(m \geq n)
$$

with a logarithmic precision satisfying (5). Moreover, by properties I and III of the function $C(z)$, since $\beta_{1}>\pi j D^{*}$ and $\beta_{2}>\pi j D^{*}$, the function $f^{*}(z)$ satisfies the hypotheses of Lemma 2 , with $\beta_{1}^{*}>\pi D^{*}$ and $\beta_{2}^{*}>\pi D^{*}$. The conclusion of Lemma 2 then states that $F^{*}(s)$ can be continued analytically throughout a strip $|t| \leq \pi R$, for some $R>D^{\circ}$, in which it is uniformly bounded. It follows, then, from conclusion (6) of Theorem $1\left(-\sigma_{1}\right.$ arbitrarily large $)$ that $a_{n}^{*}=0$, hence $a_{n,-p}=0$ (Theorem $\left.3 \mathrm{~A}\right)$ or $a_{n, p}=0$ (Theorem 3B) $(n=1,2, \cdots)$. Successive applications of this result show that under the hypotheses of Theorem 3B, $f(z) \equiv 0$; this completes the proof of Theorem 3B. Successive applications to the function in Theorem $3 \mathrm{~A}$ show that $a_{n,-k}=0,1 \leq k \leq p$, so that $f(z)$ is holomorphic in $x>0$. If then in addition 


$$
\lim _{n \rightarrow \infty} \frac{n}{\lambda_{n}}=D>0
$$

Theorem 3B applies, and $f(z) \equiv 0$. This completes the proof of Theorem 3A.

If $f(z)$ is holomorphic in $x \geq 0$, and $f(z)=O\left(e^{\alpha|z|}\right)$ as $|z| \rightarrow \infty$ for some $\alpha>0$ and for $x>0$, then $f(z)$ satisfies the hypotheses of Theorem 2 with constants $M_{n}=K \exp \left(\alpha \lambda_{n}\right)$ for some constant $K$; this sequence $\left\{M_{n}\right\}$ clearly has property $(U)$. If $f$ is such a function, and

$$
p=1, \lambda_{n}=n, \text { and } \quad c_{1}=-\beta_{1}=-\beta_{2},
$$

then Theorem 3B yields as a special case a theorem of Carlson (cf. [8, p. 186]). Theorem $3 \mathrm{~B}$ contains also a more general result noted by Boas $[2, \mathrm{p} .844]$ as a consequence of Carleman's theorem. Both assume that $f(z)$ has zeros at points $z=\lambda_{n}$, and that $f(z)$ is of exponential type in the entire half-plane $x>0$. The hypotheses on the sequence $\left\{\lambda_{n}\right\}$ of the last-mentioned result are essentially the same as in Theorem $3 \mathrm{~B}$.

We observe that Theorems $3 \mathrm{~A}$ and $3 \mathrm{~B}$ may be interpreted as relating the growth of $f$ on the $y$-axis to the maximum order of poles, or minimum order of zeros, of $f$ at points $\lambda_{n}$ sufficient to imply $f \equiv 0$. In this connection it is interesting to note that Theorem 3B contains also a result of Boas in which $f(z)$ is assumed to have zeros of order 2 at points $z=\lambda_{n}=2 n$. This latter result Boas generalized in a different direction [3]. Fuchs [4], retaining the hypotheses that $f(z)$ is of exponential type throughout the half-plane $x>0$ and has zeros at points $z=\lambda_{n}$, used Carleman's theorem and properties of the function

$$
H(z)=\prod_{k=1}^{\infty}\left(\lambda_{k}-z\right) \frac{\exp \left(2 z / \lambda_{k}\right)}{\lambda_{k}+z}
$$

to give conditions on the sequence $\left\{\lambda_{n}\right\}$ which are necessary and sufficient in order that the only function satisfying these hypotheses shall vanish identically. Professor S. Mandelbrojt has kindly called to the author's attention recent work of Kahane [5] who has further generalized Carlson's theorem.

At the suggestion of the referee, the special case of Theorem $3 \mathrm{~A}$ corresponding to Carlson's theorem is stated as a corollary of Theorem 3A.

COROLlaRY 3A. If $f(z)$ is holomorphic in $x \geq 0$, except for poles of maximum order $p$ at points $z=n(n=1,2, \ldots)$ on the xaxis, if $f(z)=O\left(e^{\alpha|z|}\right)$ 
in $x>0$ for some real number $\alpha$, and $f(i y)=O\left(e^{-\beta|y|}\right)$ as $|y| \longrightarrow \propto$ for some positive number $\beta>\pi p$, then $f(z) \equiv 0$.

\section{REFERENCES}

1. V. Bernstein, Séries de Dirichlet, Gauthier-Villars, Paris, 1933.

2. R. P. Boas, Entire functions of exponential type, Bull. Amer. Math. Soc. 48 (1942), 839-849.

3. Some uniqueness theorems for entire functions, Amer. J. Math. 62 (1940), 319-324.

4. W. H. J. Fuchs, A generalization of Carlson's theorem, J. London Math. Soc. 21 (1946), 106- 110.

5. J. -P. Kahane, Extension du theoreme de Carlson et applications, C. R. Acad. Sci. Paris 234 (1952), 2038-2040.

6. S. Mandelbrojt, Théorèmes d'unicité, Ann. Sci. Ecole Norm. Sup. (3) 65 (1948), $101-138$.

7. - Séries adhérentes. Regularisation des suites. Applications. GauthierVillars, Paris, 1952.

8. E. C. Titchmarsh, The the ory of functions, Oxford, 1932.

UNIVERSITY OF MisSOURI 



\title{
PACIFIC JOURNAL OF MATHEMATICS
}

\section{EDITORS}

\author{
M. M. SCHIFFER* \\ Stanf ord University \\ Stanf ord, California \\ E. HewitT \\ University of Washington \\ Seattle 5, Washington
}

\author{
P. P. DILWCR TH \\ California Institute of Technology \\ Pasadena 4, California \\ E. F. BECKENBACH** \\ University of California \\ Los Angeles 24, California
}

\section{ASSOCIATE EDITORS}

$\begin{array}{ll}\text { H. BUSEMANN } & \text { P. R. HALMOS } \\ \text { HERDERT FF.DERER } & \text { HEINZ HOPF } \\ \text { MARSH ALLL IHALL } & \text { R. D. JAMES }\end{array}$

MARSH AL.L II ALL

\author{
R. D. JAMES
}
BORGE JESSEN
PAUL LÉVY
GEORGE PÓLYA

\author{
J. J. STOKER \\ E. G. STRAUS
}
KÖSAKU YOSIDA

\section{SPONSORS}

UNIVERSITY OF BRITISH COLUMBIA CAI IFORNIA ENSTITUTE OF TECHNOLOGY UNIVERSITY OF CALIFORNIA, BERKELEY UNIVERSITY OF CALIFORNIA, DAVIS UNIVERSITY OF CALIFORNIA, LOS ANGELES UNIVERSITY OF CALIFORNI A, SANTA BARBARA UNIVERSITY OF NEVADA OREGON STATE COLLEGE UNIVERSITY OF OREGON
UNIVERSITY OF SOU THERN CALIFORNIA STANFORD RESE.ARCH INSTITUTE STANFORD UNIVERSITY WASHING TON STATE COLLEGE UNIVERSITY OF WASHINGTON

AMERICAN MATHEMATICAL SOCIETY HUGHES AIRCRAFT COMPANY SHELL DEVELOPMENT COMPANY

* To be succeeded in 1955, by H.L. Royden, Stanford University, Stanford, California.

${ }^{* *}$ To be succeeded in 1955, by E.G. Straus, University of California, Los Angeles 24, Calif.

\author{
Vari-Type Composition by \\ Elaine Barth
}

Printed in the United States of America by

Edwards Brothers, Inc., Ann Arbor, Michigan

UNIVERSITY OF CALIFORNIA PRESS * BERKELEY AND LOS ANGELES COPYRIGHT 1954 BY PACIFIC JOURNAL OF MATHEMATICS 


\section{Pacific Journal of Mathematics}

\section{Vol. 4, No. 1 \\ May, 1954}

Hugh D. Brunk, On the growth of functions having poles or zeros on the positive real axis ................................. 1

J. Copping, Application of a theorem of Pólya to the solution of an infinite

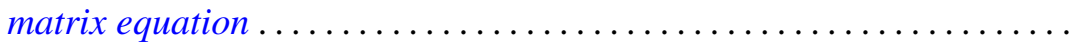

James Richard Jackson, On the existence problem of linear programming ................................... 29

Victor Klee, Invariant extension of linear functionals ............... 37

Shu-Teh Chen Moy, Characterizations of conditional expectation as a transformation on function spaces ..................... 47

Hukukane Nikaidô, On von Neumann's minimax theorem .............. 65

Gordon Marshall Petersen, Methods of summation .................. 73

G. Power, Some perturbed electrostatic fields .................. 79

Murray Harold Protter, The two noncharacteristic problem with data partly

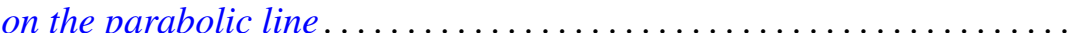

S. E. Rauch, Mapping properties of Cesàro sums of order two of the geometric series........................................... 109

Gerson B. Robison, Invariant integrals over a class of Banach spaces . . . . 123

Richard Steven Varga, Eigenvalues of circulant matrices .............. 151 